\title{
System Reliability Evaluation Based on Convex Combination Considering Operation and Maintenance Strategy
}

\author{
Lijie Li, ${ }^{1,2}$ Limin Jia, ${ }^{1,2}$ and Yanhui Wang ${ }^{1,2}$ \\ ${ }^{1}$ State Key Laboratory of Railway Traffic Control \& Safety, Beijing Jiaotong University, Beijing 100044, China \\ ${ }^{2}$ School of Traffic Transportation, Beijing Jiaotong University, Beijing 100044, China \\ Correspondence should be addressed to Limin Jia; jialm@vip.sina.com
}

Received 27 June 2015; Revised 2 October 2015; Accepted 13 October 2015

Academic Editor: Shinji Tsujikawa

Copyright (c) 2015 Lijie Li et al. This is an open access article distributed under the Creative Commons Attribution License, which permits unrestricted use, distribution, and reproduction in any medium, provided the original work is properly cited.

\begin{abstract}
The approaches to the system reliability evaluation with respect to the cases, where the components are independent or the components have interactive relationships within the system, were proposed in this paper. Starting from the higher requirements on system operational safety and economy, the reliability focused optimal models of multiobjective maintenance strategies were built. For safety-critical systems, the pessimistic maintenance strategies are usually taken, and, in these cases, the system reliability evaluation has also to be tackled pessimistically. For safety-uncritical systems, the optimistic maintenance strategies were usually taken, and, in these circumstances, the system reliability evaluation had also to be tackled optimistically, respectively. Besides, the reasonable maintenance strategies and their corresponding reliability evaluation can be obtained through the convex combination of the above two cases. With a high-speed train system as the example background, the proposed method is verified by combining the actual failure data with the maintenance data. Results demonstrate that the proposed study can provide a new system reliability calculation method and solution to select and optimize the multiobjective operational strategies with the considerations of system safety and economical requirements. The theoretical basis is also provided for scientifically estimating the reliability of a high-speed train system and formulating reasonable maintenance strategies.
\end{abstract}

\section{Introduction}

With the rapid development of Chinese high-speed trains, traditional maintenance strategies may not fit the train organization pattern of "high density, high frequency, high security" due to its disadvantages of short overhaul period, high maintenance costs, and long parking time. The train maintenance work will turn to the trend of "reliabilitycentered maintenance."

Numerous researches focused on this topic. Jin et al. [1] proposed the optimization of reliability-centered nonperiodic preventive maintenance (PM) for multicomponent equipment with minimal repair at failures. Three types of PM actions including mechanical service, repair, and replacement were simultaneously considered. Eti et al. [2] summarized the research process that the center of maintenance strategy changed from the reactive repair-focused pattern to the reliability-centered pattern. Faza et al. proposed a quantitative approach to modeling the reliability of the advanced power grid [3]. Zhou et al. [4] integrated the sequential imperfect maintenance policy into condition-based predictive maintenance, and a reliability-centered predictive maintenance policy was proposed for a continuously monitored system subject to degradation due to the imperfect maintenance. He et al. [5] found out that operational reliability evaluation theory reflected real-time reliability level of power system, and the component failure rate varied with operating conditions.

The results from above-mentioned studies contribute significantly to the development and investigation of the relationship between maintenance strategy and reliability. But they were difficult to be applied in the area of high-speed train systems. On the one hand, these studies mainly focused on the relatively simple systems, whereas the high-speed train system is a complex system. On the other hand, traditional reliability evaluation methods, such as Fault Tree, Bayesian Network, Markov, and Petri Nets, always assumed that the components are independent. For example, a comprehensive 


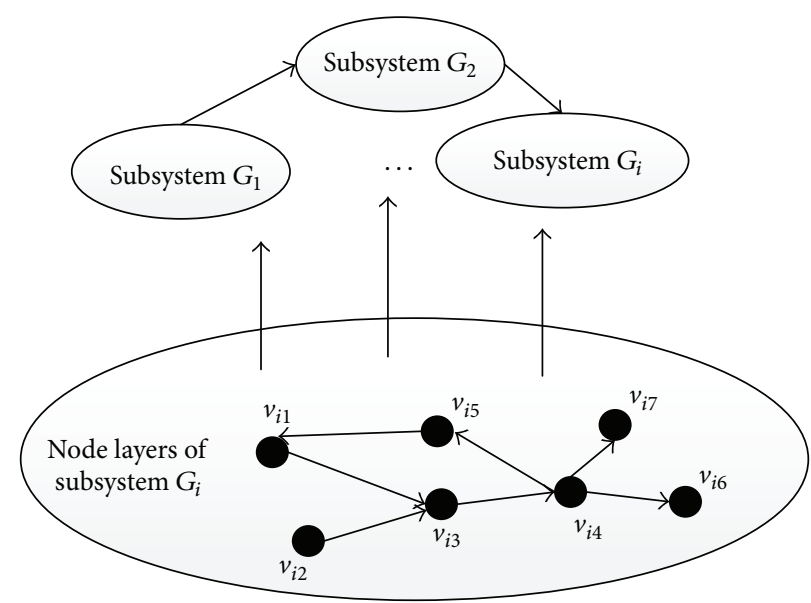

FIGURE 1: Model of system reliability consisted of independent components.

software safety analysis involving a combination of Failure Modes and Effects Analysis (FMEA) and Fault Tree Analysis (FTA) is conducted on the software functions of the critical system to identify potentially hazardous software faults [6]. Sarhan and El-Gohary [7] estimated the parameters of reliability model by maximum likelihood and Bayesian methods. A semi-Markov process with four states has been applied for modeling two dissimilar unit cold standby systems [8]. Petri Nets have been applied to the field of mechanical system reliability analysis [9]. In fact, there were quite complicated relationships among components in the system. That is why the results obtained by traditional methods were always far away from the actual situation.

The system reliability evaluation approaches for the cases, where the components are independent or the components have interactive relationships within the system, are proposed in this paper. Starting from the higher requirements on system operational safety and cost-effectiveness, the reliability focused optimal models of multiobjective maintenance strategies are built. System reliability evaluation is accomplished under different maintenance strategies. Finally, with a high-speed train system as an example background, the proposed method is verified by combining the actual failure data with the maintenance data.

\section{Reliability Modeling and Calculation for the System with Independent Components}

2.1. Reliability Network Modeling for the System with Independent Components. The following hypotheses are made when building reliability models on the system with independent components.

Hypothesis 1. Components (nodes) are independent.

Hypothesis 2. There are only two states: normal and fault.

Hypothesis 3. Edges are independent, which means the failure of one single edge does not affect the others.
A two-tuple group $\langle V, E\rangle$ was built, and, assuming $V$ is nonempty, $V=\left\{v_{1}, v_{2}, \ldots, v_{n}\right\}$, which can be called components set. $E$ is a subset which belongs to $V \times V$, and $E=$ $\left\{e_{1}, e_{2}, \ldots, e_{n}\right\}$ is called edges set. The edge $e_{i j}$ in the directed graph is an ordered pair that consists of two vertexes, and the ordered pair is often represented by angle brackets; for instance, $\left\langle v_{i}, v_{j}\right\rangle$ represents a directed edge, $v_{i}$ is the starting point of the directed edge, and $v_{j}$ is the finishing point. $\left\langle v_{i}, v_{j}\right\rangle$ and $\left\langle v_{j}, v_{i}\right\rangle$ are two different directed edges, as shown in Figure 1 .

\subsection{Reliability Calculation for the System with Independent Components}

2.2.1. Series System. It is supposed that system $G$ consists of $n$ units components in a series way, which means the whole system will be failed when any component of the system is invalid, as shown in Figure 2. The lifetime of $i$ component is $T_{i}$, the operational reliability is $R_{i}(t)$, and the lifetime distribution function is $F_{i}(t), i=1,2, \ldots, n$; then the lifetime of system $G$ is $T=\min \left(T_{1}, T_{2}, \ldots, T_{n}>t\right)$.

Assuming $T_{1}, T_{2}, \ldots, T_{n}$ are independent, the reliability of the series system is

$$
\begin{aligned}
R_{0}(t) & =P\left\{\min \left\{T_{1}, T_{2}, \ldots, T_{n}\right\}>t\right\} \\
& =P\left\{T_{1}>t, T_{2}>t, \ldots, T_{n}>t\right\}=\prod_{i=1}^{n} P\left\{T_{i}>t\right\} \\
& =\prod_{i=1}^{n} R_{i}(t) .
\end{aligned}
$$

The mean life of the system is

$$
\operatorname{MTTF}=\int_{0}^{\infty} R_{0}(t) d t
$$

2.2.2. Parallel System. It is supposed that system $G$ consists of $n$ units components in a parallel way, which means that only 


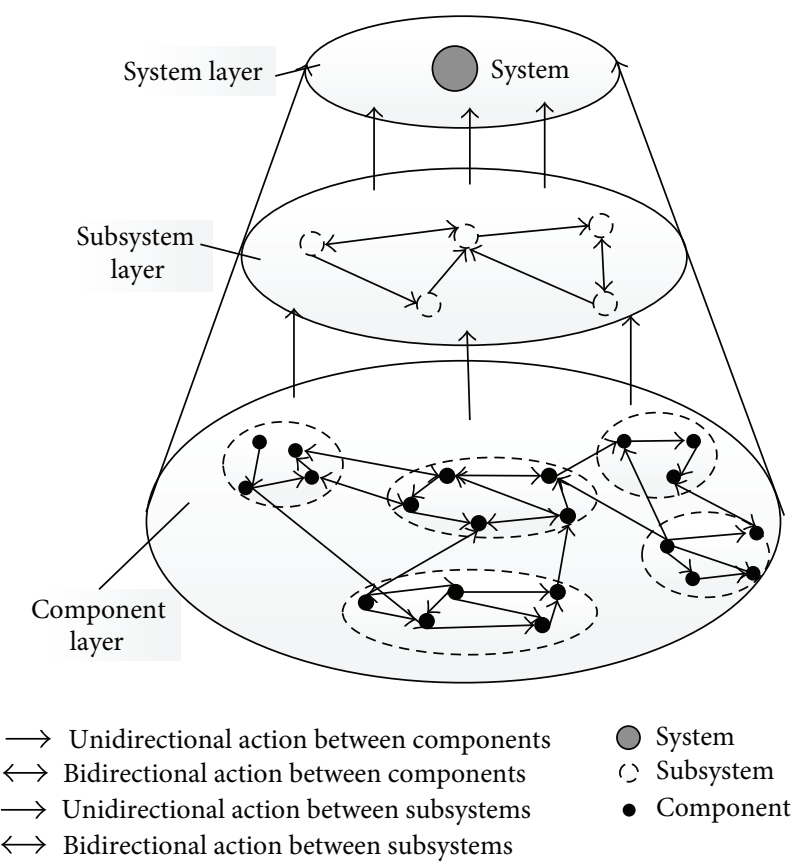

FIGURE 2: Reliability network modeling on the system with dependent components.

when $n$ components are all failed will the whole system be invalid.

The lifetime of $i$ component is $T_{i}$, the operational reliability is $R_{i}(t)$, and the lifetime distribution function is $F_{i}(t), i=1,2, \ldots, n$; then the lifetime of system $G$ is $T=\max \left(T_{1}, T_{2}, \ldots, T_{n}>t\right)$. Assuming $T_{1}, T_{2}, \ldots, T_{n}$ are independent of each other, the reliability of the parallel system is

$$
\begin{aligned}
R_{0}(t) & =P\left\{\max \left\{T_{1}, T_{2}, \ldots, T_{n}\right\}>t\right\} \\
& =1-P\left\{T_{1} \leq t, T_{2} \leq t, \ldots, T_{n} \leq t\right\} \\
& =1-\prod_{i=1}^{n}\left(1-R_{i}(t)\right) .
\end{aligned}
$$

\section{Reliability Modeling and Calculation for the System with Dependent Components}

3.1. Reliability Network Modeling for the System with Dependent Components. The following hypotheses are assumed for building reliability models of the system with dependent components.

Hypothesis 1. The system topology structure is fixed.

Hypothesis 2. There are only two states in the components: normal and fault.

Hypothesis 3. If the functional relationship of the parts of the system is normal, the state of the subsystem is normal, or it is in the failure state.
Hypothesis 4. In the system reliability models, the failure of nodes is equivalent to the failure of the functional relationship among the nodes.

There are two ways to express the functional relationship among the parts, as shown in Table 1.

In the study of subsystem reliability, the system components were set as nodes, while in the study of system reliability, the subsystems were set as nodes, and the functional relationship between nodes was edge. Directed two-layer network models were built with characteristics of network topology structure.

3.1.1. Network Models on Components Layer. In the study of subsystem reliability, each subsystem consists of a series of indivisible components. The study sets the components as nodes, the operational reliability of components as the nodes properties, and the functional relationship between components as edges to build a directed network model with characteristics of network topology structure, $G_{i}=(v, e, r)$. The specific explanations are given as follows:

$v$-collection of nodes, $v\left(G_{i}\right)=\left\{v_{i 1}, v_{i 2}, \ldots, v_{i n}\right\}$, which is the collection of components.

$e$-collection of edges, $e\left(G_{i}\right)=\left\{e_{i 1}, e_{i 2}, \ldots, e_{i n}\right\}$, which is the collection of functional relationships between components.

$r$-collection of the nodes properties, namely, the operational reliability of components, and $r_{i j}(t)(i=$ $1,2, \ldots, \varepsilon ; j=1,2, \ldots, s)$ represents the reliability of the component $j$ in subsystem $G_{i}$ at time $t$. 
TABLE 1: The way of connection between the components and its formalized expression.

\begin{tabular}{|c|c|c|}
\hline Ways of connection & Graphical representation & Description \\
\hline Unidirectional connection & $\mathrm{O} \longrightarrow 0$ & One-way functional relationship between two components \\
\hline Bidirectional connection & 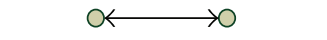 & The interaction between two components \\
\hline
\end{tabular}

3.1.2. Network Models on Subsystems Layer. The study of system reliability sets the system as nodes and the functional relationship between subsystems as edges, building the directed network models with characteristics of network topology structure, $G=(V, E, R)$. The specific explanations are given as follows:

$V$-collection of nodes, $V(G)=\left\{G_{1}, G_{2}, \ldots, G_{m}\right\}$, which is the collection of subsystems.

E-collection of edges, which is the collection of functional relationships between subsystems.

$R$-collection of the nodes properties, which is the collection of reliability of subsystems, $R(t)=$ $\left\{R_{1}(t), R_{2}(t), \ldots, R_{m}(t)\right\}, R_{i}(t)$ representing the reliability of subsystem $G_{i}$ at time $t$.

3.2. Reliability Calculation for the System with Dependent Components Based on Copula Function. In order to calculate the reliability of systems which consisted of dependent components, Schweizer and Sklar [10] put forward the Copula Function. The Copula Function describes the correlation between variables. Copula is a function that combines the distribution functions with their corresponding edge distribution functions together. This paper calculates the reliability of the system with dependent components from two aspects (series system and parallel system) based on Copula Function.

3.2.1. Reliability of Series System Based on Copula. It is assumed that system $G$ consists of $n$ units components in a series way, the lifetime of the $i$ component is $T_{i}$, the operational reliability is $R_{i}(t)$, and the lifetime distribution function is $F_{i}(t), i=1,2, \ldots, n$; then the lifetime of the system $G$ is $T=\min \left(T_{1}, T_{2}, \ldots, T_{n}>t\right)$. The joint distribution function is $H\left(t_{1}, t_{2}, \ldots, t_{n}\right)=P\left\{T_{1} \leq t_{1}, T_{2} \leq t_{2}, \ldots, T_{n} \leq t_{n}\right\}$. According to the Sklar lemma, there is an $n$-dimensional Copula $C$ making $H\left(t_{1}, t_{2}, \ldots, t_{n}\right)=C\left(F_{1}\left(t_{1}\right), F_{2}\left(t_{2}\right), \ldots, F_{n}\left(t_{n}\right)\right)$; $C\left(F_{1}\left(t_{1}\right), F_{2}\left(t_{2}\right), \ldots, F_{n}\left(t_{n}\right)\right)$ is unique, because $F_{i}(t)$ is continuous. The degree of the reliability of system $G$ is shown as follows:

$$
\begin{gathered}
R_{c}(t)=P\left\{\min \left(T_{1}, T_{2}, \ldots, T_{n}\right)>t\right\}=P\left(T_{1}>t, T_{2}\right. \\
\left.>t, \ldots, T_{n}>t\right)=1-\sum_{i=1}^{n} P\left(T_{i} \leq t\right)+\sum_{1 \leq i \leq j \leq n} P\left(T_{i}\right. \\
\left.\leq t, T_{j} \leq t\right)+\cdots+(-1)^{k} \sum_{1<i_{1}<i_{2}<\cdots<i_{k} \leq n} P\left(T_{i_{1}} \leq t, T_{i_{2}}\right. \\
\left.\quad \leq t, \ldots, T_{i_{k}} \leq t\right)+\cdots+(-1)^{n} P\left(T_{i_{1}} \leq t, T_{i_{2}} \leq t, \ldots,\right.
\end{gathered}
$$

$$
\begin{aligned}
& \left.T_{i_{k}} \leq t\right)=1-\sum_{i=1}^{n} F_{i}(t)+(-1)^{k} \sum_{1<i_{1}<i_{2}<\cdots<i_{k} \leq n} C\left(F_{i_{1}}(t),\right. \\
& \left.F_{i_{2}}(t), \ldots, F_{i_{k}}(t)\right)=1-\sum_{i=1}^{n} F_{i}(t)+(-1)^{k} \\
& \cdot \sum_{1<i_{1}<i_{2}<\cdots<i_{k} \leq n} C\left(F_{i_{1}}(t), F_{i_{2}}(t), \ldots, F_{i_{k}}(t),\right. \\
& \underbrace{1, \ldots, 1}_{\text {rest } n-k}) \quad(2 \leq k \leq n) .
\end{aligned}
$$

3.2.2. The Reliability of Parallel System Based on Copula. It is assumed that system $G$ consists of $n$ units components in a parallel way, the lifetime of $i$ component is $T_{i}$, the operational reliability is $R_{i}(t)$, the lifetime distribution function is $F_{i}(t), i=1,2, \ldots, n$, and the lifetime of system $G$ is $T=\max \left(T_{1}, T_{2}, \ldots, T_{n}\right)$. The joint distribution function is $H\left(t_{1}, t_{2}, \ldots, t_{n}\right)=P\left\{T_{1} \leq t_{1}, T_{2} \leq t_{2}, \ldots, T_{n} \leq t_{n}\right\}$. According to the Sklar lemma [10], there is an $n$-dimensional Copula $C$ making $H\left(t_{1}, t_{2}, \ldots, t_{n}\right)=C^{n}\left(F_{1}\left(t_{1}\right), F_{2}\left(t_{2}\right), \ldots, F_{n}\left(t_{n}\right)\right)$; $C^{n}\left(F_{1}\left(t_{1}\right), F_{2}\left(t_{2}\right), \ldots, F_{n}\left(t_{n}\right)\right)$ is unique because $F_{i}(t)$ is continuous. The degree of the reliability of the system $G$ is shown as follows:

$$
\begin{aligned}
R_{c}(t) & =P\left\{\max \left(T_{1}, T_{2}, \ldots, T_{n}\right)\right\} \\
& =1-P\left\{\max \left(T_{1}, T_{2}, \ldots, T_{n}\right) \leq t\right\} \\
& =1-C F_{1}(t), F_{2}(t), \ldots, F_{n}(t) \\
& =1-C\left(1-R_{1}(t), 1-R_{2}(t), \ldots, 1-R_{n}(t)\right) .
\end{aligned}
$$

\section{System Reliability Analysis Based on Different Connecting Types}

Considering the fact that this study calculates the system reliability based on the connectivity of the system inside components, the system reliability may be affected by the connecting type of system components.

The calculating methods of system reliability are discussed in different connecting types in this paper. General system connecting types mainly include three ways: single input and single output, single input and multiple outputs, and multiple inputs and multiple outputs (multiple inputs and single output connecting type is the same as single input and multiple outputs connecting type).

4.1. Single Input and Single Output. As shown in Figure 3, it describes how to calculate the reliability of subsystem $G_{1}$ in 


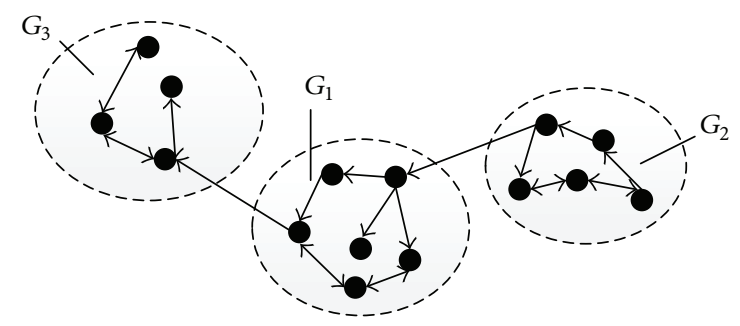

Figure 3: Model of single input and single output.

the situation of single input node and single output node. According to the network models, supposing that system $G$ consists of $m$ subsystems, $G=\left\{G_{1}, G_{2}, \ldots G_{m}\right\}$, subsystem $G_{q}$ inputs node $v_{q s}$ and outputs node $v_{q l}$; the reliability calculation of subsystem $G_{q}$ can be converted into the analysis of probability of node $v_{q s}$ to node $v_{q l}$; that is,

$$
R_{q s l}=P\left\{v_{q s} \text { can reach } v_{q s l}\right\} \text {. }
$$

All the shortest paths should be figured out among the subsystems to analyze the reliability of subsystem based on the minimal path set. It is supposed that $d_{1}, d_{2} \ldots, d_{n d}$ are all shortest paths from node $v_{q s}$ to node $v_{q l}$, and $n d$ represents the quantity of minimal paths:

(1) $n d=1$; all nodes on the minimal path are in one series. For the subsystem, it will be in a normal state when all nodes on the minimal path are normal. Therefore the reliability of the minimal path is the reliability of subsystem.

(2) $n d>1$; it is supposed that $d_{i}, i=1,2, \ldots, n d$, represents any minimal path; the reliability of the minimal path $d_{i}$ is the same as that of all nodes on path $d_{i}$ work well. For the subsystem, it will work properly only if one of the minimal paths was in normal state, which means the highest reliability of the minimal path can be viewed as the reliability of the subsystem.

In conclusion, no matter how many minimal paths are there in the subsystem, the highest reliability of nd minimal path ( $n d=1$, only need to solve the unique minimal path) can be considered as the reliability of the subsystem:

$$
R(t)=\max \left(R\left(d_{i}\right)\right), \quad i=1,2, \ldots, n d .
$$

(1) System reliability is calculated with independent components.

The reliability of every minimal path based on (1) can be illustrated as follows:

$$
R_{0}\left(d_{i}\right)=\prod_{j=1}^{n} R_{j}(t)=\prod_{j=1}^{n}\left(1-F_{j}(t)\right) .
$$

$R_{0}\left(d_{i}\right)$ is the connecting reliability value of the minimal path $d_{i}$, and $F_{j}(t)$ is the lifetime distribution function of component $j$ constituting the minimal path $d_{i}$.

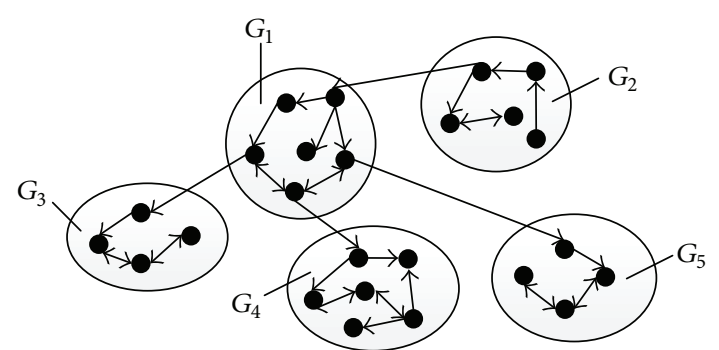

FIGURE 4: Single input and multiple outputs.

The reliability of subsystem is

$$
R_{0}\left(G_{q}\right)=\max R_{0}\left(d_{i}\right),
$$

$i=1,2, \ldots, n d ; R_{0}\left(G_{q}\right)$ is the reliability of the subsystem.

(2) System reliability is calculated with dependent components.

The reliability of every minimal path based on (4) can be illustrated as follows:

$$
\begin{aligned}
& R_{c}\left(d_{i}\right)=1-\sum_{j=1}^{n} F_{j}(t)+(-1)^{k} \\
& . \sum_{1<j_{1}<j_{2}<\cdots<j_{k} \leq n} C^{n}\left(F_{j_{1}}(t), F_{j_{2}}(t), \ldots, F_{j_{k}}(t),\right. \\
& \underbrace{1, \ldots, 1}_{\text {rest } n-k}) .
\end{aligned}
$$

$R_{c}\left(d_{i}\right)$ is the connecting reliability value of the minimal path $d_{i}$ and $F_{j}(t)$ is the lifetime distribution function of component $j$ constituting the minimal path $d_{i}$. The reliability of the subsystem $G_{q}$ is

$$
R_{c}\left(G_{q}\right)=\max R_{c}\left(d_{i}\right),
$$

$i=1,2, \ldots, n d ; R_{c}\left(G_{q}\right)$ is the reliability of subsystem $G_{q}$.

4.2. Single Input and Multiple Outputs. As shown in Figure 4, the shortest path was analyzed from input node $v_{0}$ to output nodes (supposing $n$ nodes) in subsystem $G_{1}$ based on Dijkstra's algorithm, $d_{1}, d_{2} \ldots, d_{n}$. The reliability of every minimal path is calculated according to (8) and (10).

For subsystems, the reliability of $R_{0}\left(G_{q}\right)$ and $R_{c}\left(G_{q}\right)$ can be calculated, respectively, according to (9) and (11) in the situations of independent and dependent components.

This calculation method can also calculate the reliability of subsystem with multiple input nodes and single output node; the details will not be described here.

4.3. Multiple Inputs and Multiple Outputs. As shown in Figure 5, it is supposed that the subsystem consists of $i$ input nodes, $j$ output nodes, and $C_{i}^{1} C_{j}^{1}$ node pairs (one input node and one output node). It is required to calculate the shortest path in all these node pairs. $d_{1}, d_{2} \ldots, d_{C_{i}^{1} C_{j}^{1}}$ is 


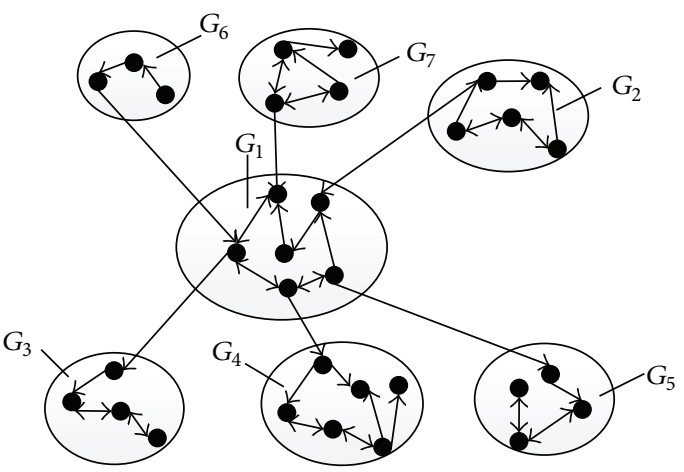

FIgURE 5: Multiple inputs and multiple outputs.

the shortest path in all these pairs based on Floyd's algorithm. The reliability of every shortest path in situations of independent and dependent components can be calculated, respectively, according to (8) and (10).

For subsystems, the reliability of $R_{0}\left(G_{q}\right)$ and $R_{c}\left(G_{q}\right)$ can be calculated, respectively, according to (9) and (11).

4.4. Summary. As can be seen from the above-mentioned calculation based on three connecting types, no matter which connecting type is, the reliability of subsystems, $R_{0}\left(G_{q}\right)$ and $R_{c}\left(G_{q}\right)$, can be calculated according to (9) and (11) under the situations of independent and dependent components. Therefore, it is unnecessary to take the connecting type of system components into consideration when calculating the system reliability using (9) and (11) directly.

\section{System Reliability Estimation}

5.1. Comparison of System Reliability Estimation between Independent Components and Dependent Components. According to comprehensive comparison and analysis on the system reliability estimation methods above, supposing that the system reliability of dependent components is greater than that of independent components and trying to verify it by using the method of Mathematical Induction, the proofs are as follows:

(1) $n=2$, which means the two nodes on the minimal path in series. is

When components are dependent, the system reliability

$$
\begin{aligned}
R_{0}\left(d_{i}\right)_{2}= & 1-P\left\{T_{1}>t, T_{2}>t\right\} \\
= & 1-P\left\{T_{1} \leq t\right\}-P\left\{T_{2} \leq t\right\} \\
& +P\left\{T_{1} \leq t, T_{2} \leq t\right\} \\
= & 1-P\left\{T_{1} \leq t\right\}-P\left\{T_{2} \leq t\right\} \\
& +\prod\left(F_{1}(t), F_{2}(t)\right) .
\end{aligned}
$$

When components are independent, the system reliability is

$$
\begin{aligned}
R_{c}\left(d_{i}\right)_{2}= & P\left\{T_{1}>t, T_{2}>t\right\} \\
= & 1-P\left\{T_{1} \leq t\right\}-P\left\{T_{2} \leq t\right\} \\
& +P\left\{T_{1} \leq t, T_{2} \leq t\right\} \\
= & 1-P\left\{T_{1} \leq t\right\}-P\left\{T_{2} \leq t\right\} \\
& +C\left(F_{1}(t), F_{2}(t)\right) .
\end{aligned}
$$

Because $C\left(F_{1}(t), F_{2}(t)\right)>\prod\left(F_{1}(t), F_{2}(t)\right)$, we have $R_{c}\left(d_{i}\right)_{2}>R_{0}\left(d_{i}\right)_{2}$.

(2) Suppose $n=k$, and $R_{c}\left(d_{i}\right)_{k}>R_{0}\left(d_{i}\right)_{k}$.

When $n=k+1$, the system reliability with independent components is obtained as

$$
\begin{aligned}
& R_{0}\left(d_{i}\right)_{k+1}=P\left\{\min \left(T_{1}, T_{2}, \ldots, T_{k}\right)>t, T_{k+1}>t\right\} \\
&= P\left(\min \left(T_{1}, T_{2}, \ldots, T_{k}\right)>t\right) \\
& \quad-P\left\{\min \left(T_{1}, T_{2}, \ldots, T_{k}\right)>t, T_{k+1} \leq t\right\} \\
&= R_{0}\left(d_{i}\right)_{k}-P\left(T_{k+1} \leq t\right) \\
&+P\left(\min \left(T_{1}, T_{2}, \ldots, T_{k}\right) \leq t\right) P\left(T_{k+1} \leq t\right) .
\end{aligned}
$$

The system reliability with dependent components is

$$
\begin{aligned}
& R_{c}\left(d_{i}\right)_{k+1}=P\left\{\min \left(T_{1}, T_{2}, \ldots, T_{k}\right)>t, T_{k+1}>t\right\} \\
&= P\left(\min \left(T_{1}, T_{2}, \ldots, T_{k}\right)>t\right) \\
&-P\left\{\begin{array}{c}
\min \left(T_{1}, T_{2}, \ldots, T_{k}\right)>t \\
T_{k+1} \leq t
\end{array}\right\} \\
&> R_{c}\left(d_{i}\right)_{k}-P\left(T_{k+1} \leq t\right) \\
&+P\left(\min \left(T_{1}, T_{2}, \ldots, T_{k}\right) \leq t, T_{k+1} \leq t\right) \\
&> R_{c}\left(d_{i}\right)_{k}-P\left(T_{k+1} \leq t\right) \\
&+P\left(\min \left(T_{1}, T_{2}, \ldots, T_{k}\right) \leq t\right) P\left(T_{k+1} \leq t\right) \\
&= R_{0}\left(d_{i}\right)_{k+1} .
\end{aligned}
$$

The hypotheses are supported, $R_{c}\left(d_{i}\right)>R_{0}\left(d_{i}\right)$, and the system reliability of dependent components is surely greater than that of independent components.

\subsection{System Reliability Estimation Based on \\ Convex Combination Considering Operation and \\ Maintenance Strategy}

5.2.1. Estimation Method. According to Section 5.1, the system reliability of dependent components is greater than that of independent components; namely, these two estimation methods compose the upper limit $R_{c}(t)$ and lower limit $R_{0}(t)$, respectively.

Safety-critical systems should take the relatively conservative method to estimate the reliability of independent 
components, which requires using the lower limit $R_{0}(t)$ for the reliability estimation. Non-safety-critical systems should take the relatively optimistic and rational method to estimate the reliability of dependent components, setting that as the upper limit $R_{c}(t)$. For most general systems, the actual reliability is usually between the upper limit and lower limit. It will increase unnecessary maintenance cost when the reliability estimation method of independent components is used, while the maintenance cost will be reduced by taking the reliability estimation method of dependent components; however, the whole safety of the system will decrease apparently.

From the relationship balance of operational safety and economic efficiency, this paper introduces the accommodation coefficient of system operational strategy, $\lambda$. The reliability of general system in two extreme convex combinations above forms estimations based on operational strategy which is

$$
R(t)=\lambda R_{0}(t)+(1-\lambda) R_{c}(t)
$$

$\lambda$ is the accommodation coefficient of system operational strategy, and it is determined by the system itself. It is closely related to the maintenance resource, and different operational strategy corresponds to different operational resource.

5.2.2. Physical Interpretation of $\lambda$. The two extreme states of $\lambda$ are explained, respectively. Also $\lambda=1$; that is, the reliability of system is $R_{0}(t)$; using the reliability calculating method of independent components suits safety-critical systems. $\lambda=$ 0 with the reliability of system equal to $R_{c}(t)$; using the reliability calculating method of dependent components suits non-safety-critical systems. For general system, the range of value of $\lambda$ is from 0 to 1 . Each $\lambda$ corresponds to an operational strategy which contains both relatively cautious and optimistic parts.

In general, system operational strategy is a combination of the above-mentioned two extreme strategies, and the optimal $\lambda$ can be determined by the given operational resource and guaranteed optimum reliability. There should be an optimal $\lambda$ in case of giving a maintenance resource and an operational strategy. The optimal estimation value of system reliability can be obtained based on the operational strategy. Compared with the traditional system reliability estimated value without taking the operational strategy into consideration, this estimate value considers the corresponding relationship between the system reliability and maintenance, and it can best balance the relationship between the higher safety and economy of enterprises.

\section{Application and Verification of the High-Speed Train Bogie System}

This paper takes the bogie subsystem which belongs to a high-speed train system as a research example. As shown in Figure 6, the bogie subsystem reliability conforms to the situation of Section 4. The network model of bogie subsystem reliability is shown in Figure 7.

The components' names of bogie subsystem and their serial numbers are shown in Table 2. As can be seen from
TABLE 2: Components' names and serial numbers of bogie subsystem.

\begin{tabular}{|c|c|c|c|}
\hline Node number & Component & Node number & Component \\
\hline$V_{1}$ & Coupling & $V_{8}$ & Air spring \\
\hline$V_{2}$ & Gearbox & $V_{9}$ & Draft gear \\
\hline$V_{3}$ & Axle & $V_{10}$ & Beam \\
\hline$V_{4}$ & Wheel & $V_{11}$ & Primary suspension \\
\hline$V_{5}$ & Brake disc & $V_{12}$ & Axle box body \\
\hline$V_{6}$ & Brake lining & $V_{13}$ & Side beam \\
\hline$V_{7}$ & Brake clamp & $V_{14}$ & Intensifier pump \\
\hline
\end{tabular}

Figure 6, the input node is intensifier pump $\left(v_{14}\right)$, and the output node is gearbox $\left(v_{3}\right)$. According to the calculating method mentioned in Section 5.1, only one minimal path can be found in bogie subsystem using Matlab. Consider

$$
d=v_{14} \longrightarrow v_{8} \longrightarrow v_{13} \longrightarrow v_{12} \longrightarrow v_{3}
$$

This paper analyzes the lifetime distribution of five components $\left(v_{14}, v_{8}, v_{13}, v_{12}, v_{3}\right)$ on the shortest path $d$, based on the failure data of bogie subsystem under field research and taking running kilometer as lifetime data. The research collects $50 \times 5$ groups of lifetime data and estimates the parameters by the maximum likelihood estimator based on checking the distribution types, which are obeyed by each component according to Kolmogorov-Smirnov approach (KS). The lifetime of 5 components is observed as the Weibull distribution with a 0.02 significance level. The maximum likelihood estimation results of Weibull distribution parameters are shown in Table 3.

6.1. Bogie System Reliability Estimation When Components Are Dependent. According to [11], Multiple Frank Copula Function is chosen for the studies on the reliability of bogie subsystem. Probability value can be obtained after substituting $50 \times 5$ lifetime data of 5 components in Weibull's probability density function, and the Frank Copula parameter estimation of the minimal path, $\beta=3.386478$, can be obtained by using Newton iteration after substituting probability values in Frank Copula Function. Frank Copula Function of the minimal path is

$$
\begin{aligned}
C & \left(F_{1}, F_{2}, F_{3}, F_{4}, F_{5} ; \beta\right) \\
& =-\frac{1}{3.386478} \ln \left(1+\frac{\prod_{i=1}^{5}\left(e^{-3.386478 F_{i}}-1\right)}{\left(e^{-3.386478}-1\right)^{5-1}}\right) .
\end{aligned}
$$

Substituting $\beta$ and Weibull's parameter estimation values of each component in minimal path in (11), it can be found that

$$
\begin{aligned}
& R_{c}(t)=1-\sum_{j=1}^{5} F_{j}(t)+(-1)^{k} \sum_{1<j_{1}<j_{2}<\cdots<j_{k} \leq 5} C_{1}\left(F_{j_{1}}(t),\right. \\
& F_{j_{2}}(t), \ldots, F_{j_{k}}(t), \underbrace{1, \ldots, 1}_{\text {rest } 5-k}) .
\end{aligned}
$$


TABLE 3: The maximum likelihood estimation of main components in bogie subsystem.

\begin{tabular}{lcccc}
\hline Component & Number & $\begin{array}{c}\text { The parameters estimation value of Weibull distribution } \\
\eta / \mathrm{km}\end{array}$ & $m$ & Distribution function \\
\hline$v_{14}$ & 1 & 119246.3925 & 1.5436 & $F_{1}(t)=1-e^{-[t / 119246.3925]^{1.5436}}$ \\
$v_{8}$ & 2 & 108742.4035 & 1.2458 & $F_{2}(t)=1-e^{-[t / 108742.4035]^{1.2458}}$ \\
$v_{13}$ & 3 & 476891.2461 & 1.3325 & $F_{3}(t)=1-e^{-[t / 476891.2461]^{1.3325}}$ \\
$v_{12}$ & 4 & 289653.1456 & 1.2457 & $F_{4}(t)=1-e^{-[t / 289653.1456]^{1.2457}}$ \\
$v_{3}$ & 5 & 364851.2564 & 1.4688 & $F_{5}(t)=1-e^{-[t / 364851.2564]^{1.4688}}$ \\
\hline
\end{tabular}

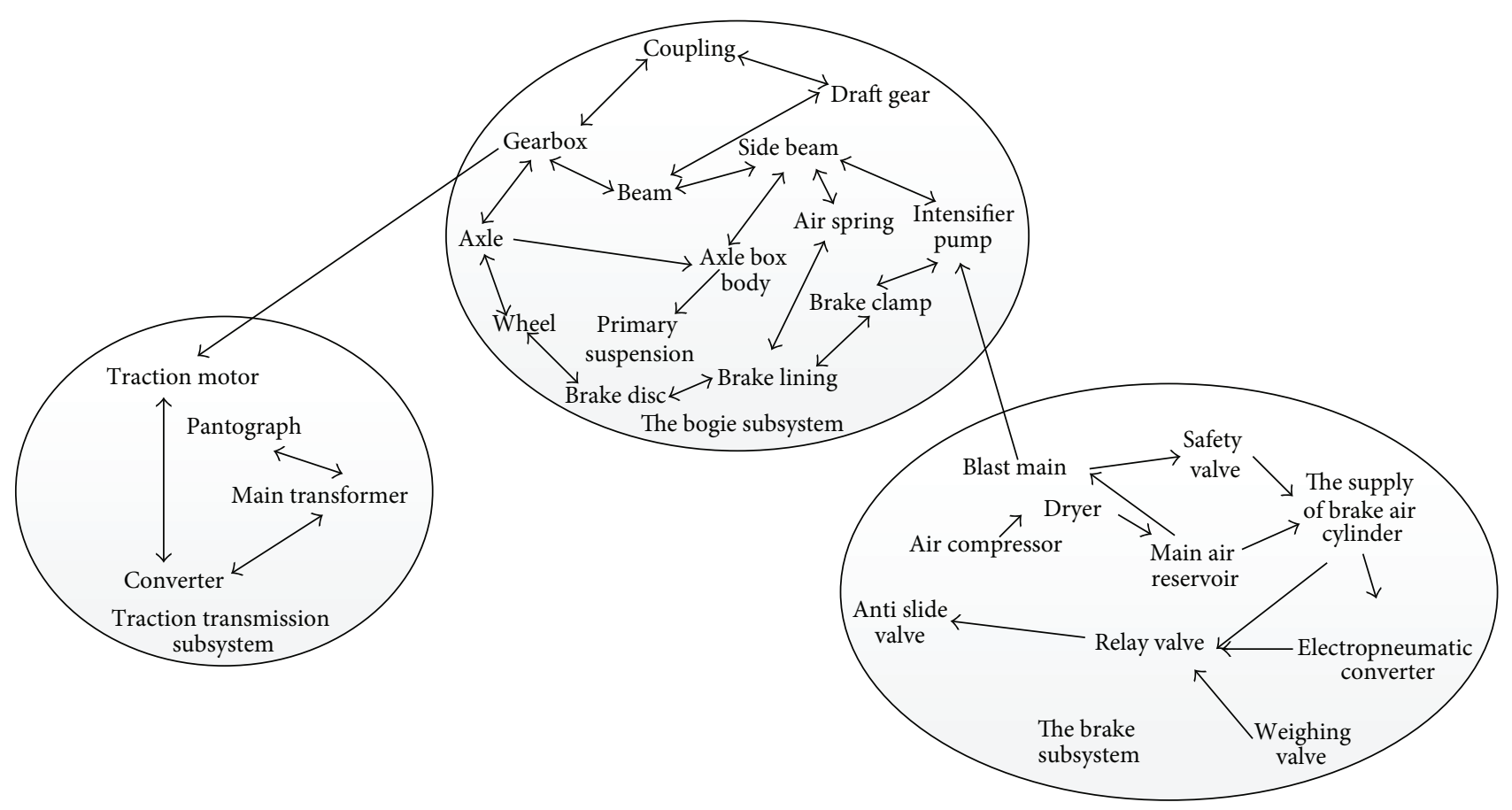

FIGURE 6: Network reliability model of the case.

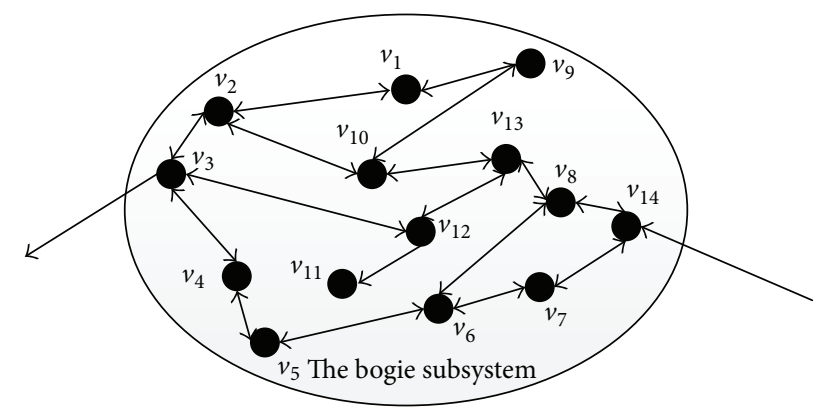

FIGURE 7: Reliability network model of bogie subsystem.

6.2. Bogie System Reliability Estimation When Components Are Independent. The subsystem reliability is $R_{0}(t)$ when supposing that nodes (components) on the minimal path are independent. It can be found that

$$
\begin{gathered}
R_{0}(t)=\prod_{i=1}^{5}\left(1-F_{i}(t)\right)=\left(1-F_{1}(t)\right)\left(1-F_{2}(t)\right) \\
\cdot\left(1-F_{3}(t)\right)\left(1-F_{4}(t)\right)\left(1-F_{5}(t)\right) .
\end{gathered}
$$

Figure 8 shows the curves comparing the reliability distribution of bogie subsystem in the above two situations. $R_{c}(t)$ represents the system reliability curve when components are dependent, and $R_{0}(t)$ represents the system reliability curve when components are independent.

The generalized expression of bogie subsystem reliability estimation is

$$
R(t)=\lambda R_{0}(t)+(1-\lambda) R_{c}(t) .
$$




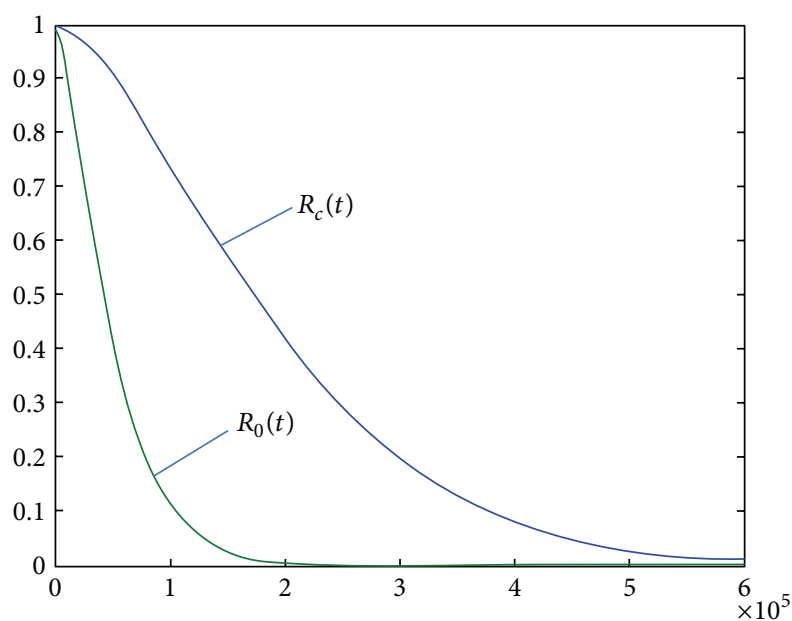

FIGURE 8: Curve graph comparing the reliability distribution of bogie subsystem based on dependent and independent components.

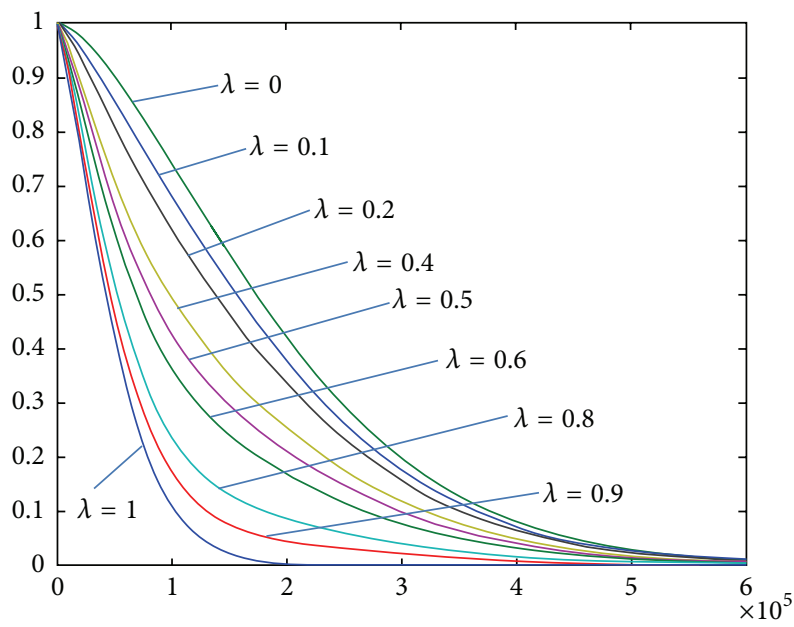

FIGURE 9: Curve graph about the bogie system reliability with different $\lambda$.

Combining with maintenance resource of the bogie system, the generalized curve of the bogie system reliability with different $\lambda$ is shown in Figure 9.

For general system, the range of $\lambda$ is from 0 to 1 . Each corresponds to an operational strategy which contains both relatively cautious and optimistic parts. For example, taking two extreme cases is shown in Figure 9. The bogie system reliability is $R_{0}(t)$ when $\lambda=1$, and $t=20000 \mathrm{~km}$, and then $R(20000)=0.7793$. The bogie system reliability is $R_{c}(t)$ when $\lambda=0$, and $t=20000 \mathrm{~km}$, and then $R(20000)=0.9755$.

It can be seen that $R_{0}(t)<R_{c}(t)$, which not only verifies that the system reliability of dependent component is greater than that of independent component but also verifies this method taking the safety and economy into consideration, and it also shows that choice and optimization of multiobjective operational strategy are rational.

\section{Discussion}

The traditional reliability theory only studies the system reliability when components are completely independent, which is too conservative and unfavorable for achieving the system potential. Considering the interaction relationship between components not only can guarantee that the system works well but also accurately reflects the reliability of whole system. Therefore, the safety property of system to accomplish tasks can be enhanced to the maximum extent.

Based on the system reliability calculation of dependent components by applied Copula Function, the convex combination considering operation and maintenance strategy was introduced. For safety-critical systems, it is suitable to estimate the system reliability based on the relatively cautious operational strategy. For non-safety-critical systems, it is suitable to estimate the system reliability based on the relatively optimistic operational strategy. For most of general system, it is better to estimate the system reliability based on the combination of the above two extreme strategies. Compared with the traditional system reliability estimate value without taking the operational strategy into consideration, this estimate value considers the corresponding relationship between the system reliability and maintenance, and it can meet the need for balance between the higher safety and economy of enterprises and also can offer a theory for scientifically estimating the system reliability of high-speed trains and making rational maintenance strategy.

Besides, with the given operational resource and guaranteed optimum reliability, the system reliability optimal estimate value can be obtained by optimizing $\lambda$. If we did related research along this thought, some better methods may be obtained to solve the issue of system reliability modeling and estimating on complex electromechanical integration.

\section{Conclusion}

This paper presents a new method to estimate the system reliability based on convex combination considering operation and maintenance strategy. The main conclusions include the following:

(1) A new thought was put forward to calculate the network system reliability of complex dependent components based on convex combination considering operation and maintenance strategy, offering a scientific and practical method to solve the issue of system reliability modeling and estimating on complex electromechanical integration.

(2) Taking the bogie system of high-speed train as the engineering background, the method proposed in this paper was applied and verified. It showed that this method can not only take the safety and economy into consideration but also obtain the rational operational strategy and realize its reliability estimation.

(3) The convex combination equation of system reliability considering operation and maintenance strategy was established in this paper. The subsequent research can 
focus on the optimization of $\lambda$ with the given operational resource and guaranteed optimum reliability.

\section{Conflict of Interests}

The authors declare that there is no conflict of interests regarding the publication of this paper.

\section{Acknowledgments}

The authors gratefully acknowledge the financial support of the projects of National Science Support Plan of China (2011BAG01802), State Key Laboratory of Rail Traffic Control and Safety (RCS2014ZT23), and CRH3 high-speed train fault data research (I11L00060).

\section{References}

[1] Y.-L. Jin, Z.-H. Jiang, and W.-R. Hou, "Optimal policy research on reliability-centered preventive maintenance for multi-components equipment," Journal of Shanghai Jiaotong University, vol. 40, no. 12, pp. 2051-2056, 2006.

[2] M. C. Eti, S. O. T. Ogaji, and S. D. Probert, "Reducing the cost of preventive maintenance (PM) through adopting a proactive reliability-focused culture," Applied Energy, vol. 83, no. 11, pp. 1235-1248, 2006.

[3] A. Faza, S. Sedigh, and B. M. McMillin, "Reliability modeling for the advanced electric power grid," in Computer Safety, Reliability, and Security: 26th International Conference, SAFECOMP 2007, Nuremberg, Germany, September 18-21, 2007. Proceedings, vol. 4680 of Lecture Notes in Computer Science, pp. 370-383, Springer, Berlin, Germany, 2007.

[4] X. Zhou, L. Xi, and J. Lee, "Reliability-centered predictive maintenance scheduling for a continuously monitored system subject to degradation," Reliability Engineering \& System Safety, vol. 92, no. 4, pp. 530-534, 2007.

[5] J. He, L. Cheng, and Y.-Z. Sun, "Transformer real-time reliability model based on operating conditions," Journal of Zhejiang University: Science A, vol. 8, no. 3, pp. 378-383, 2007.

[6] B. S. Medikonda, P. S. Ramaiah, and A. A. Gokhale, "FMEA and fault tree based software safety analysis of a railroad crossing critical system," Global Journal of Computer Science and Technology, vol. 11, no. 8, pp. 57-62, 2011.

[7] A. M. Sarhan and A. El-Gohary, "Parameter estimations of 1-out-of-2: G repairable system," Applied Mathematics and Computation, vol. 145, no. 2-3, pp. 469-479, 2003.

[8] M. Fathizadeh and K. Khorshidian, "Parameters estimation in a general failure rate semi-Markov reliability model," Journal of Statistical Theory and Applications, vol. 12, no. 3, pp. 245-252, 2013.

[9] G. Shen, C. Su, and Y. Q. Xu, "Research on the method of reliability analysis for dynamic system based on Petri Net," Mechanical Engineering \& Automation, vol. 35, no. 2, pp. 77-80, 2006.

[10] B. Schweizer and A. Sklar, "Statistical metric spaces," Pacific Journal of Mathematics, vol. 10, no. 1, pp. 313-334, 1960.

[11] C. Genest and J. MacKay, "The joy of copulas: bivariate distributions with uniform marginals," The American Statistician, vol. 40, no. 4, pp. 280-283, 1986. 


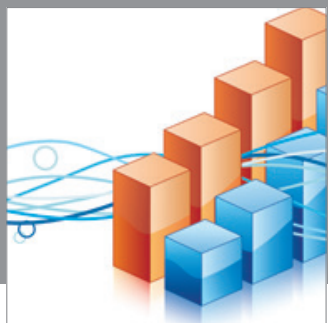

Advances in

Operations Research

mansans

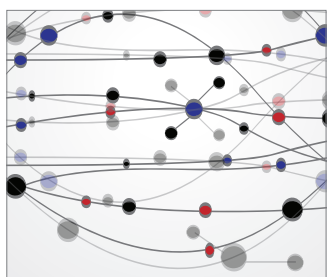

The Scientific World Journal
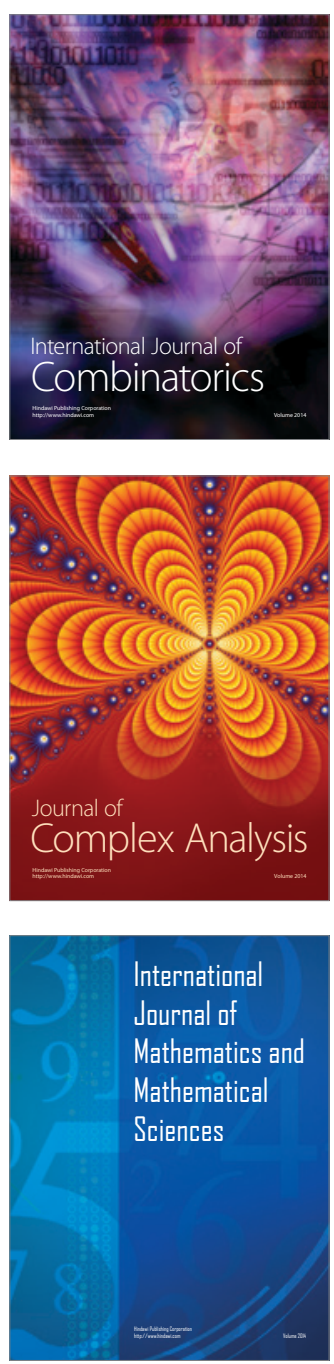
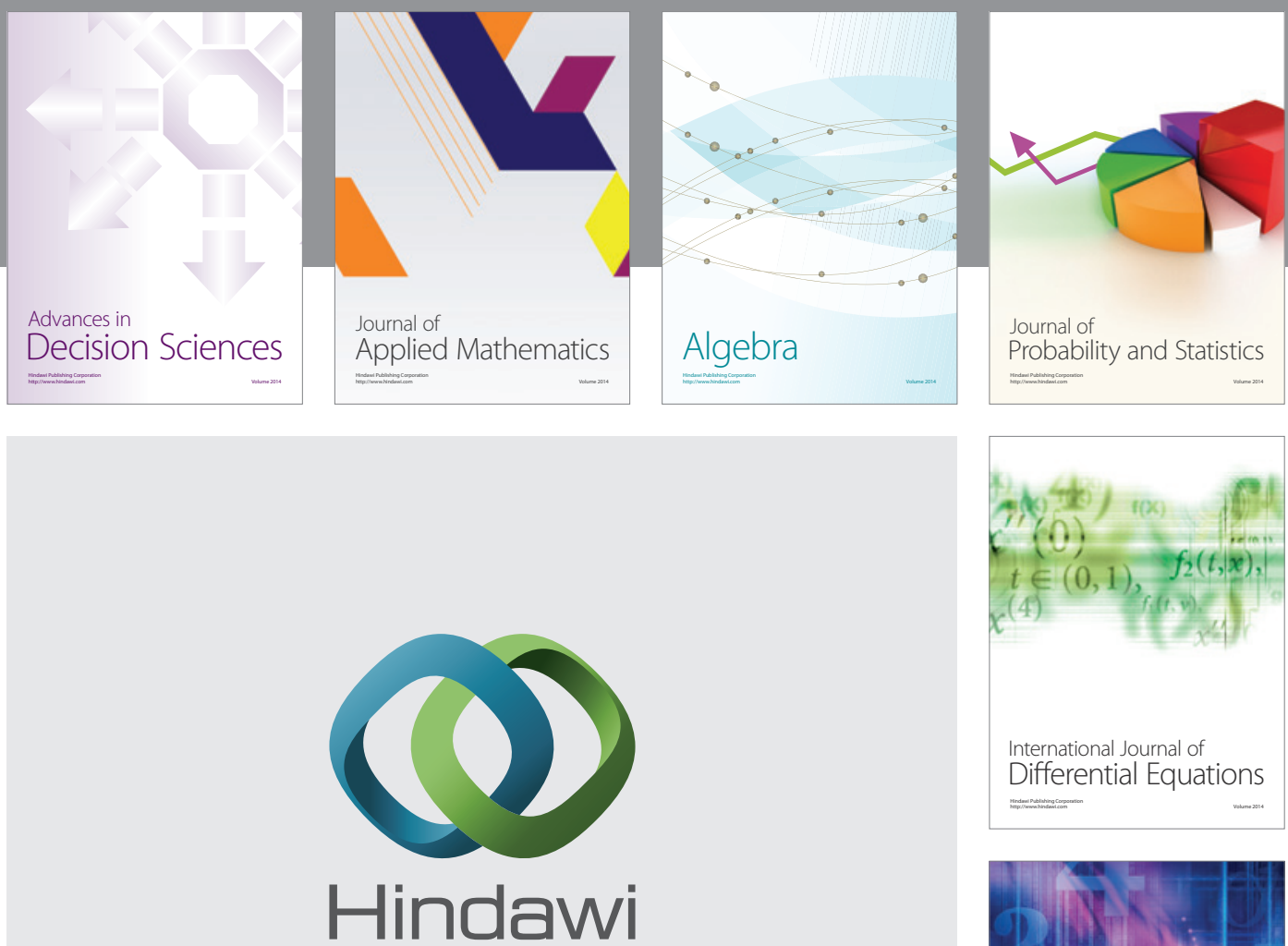

Submit your manuscripts at http://www.hindawi.com
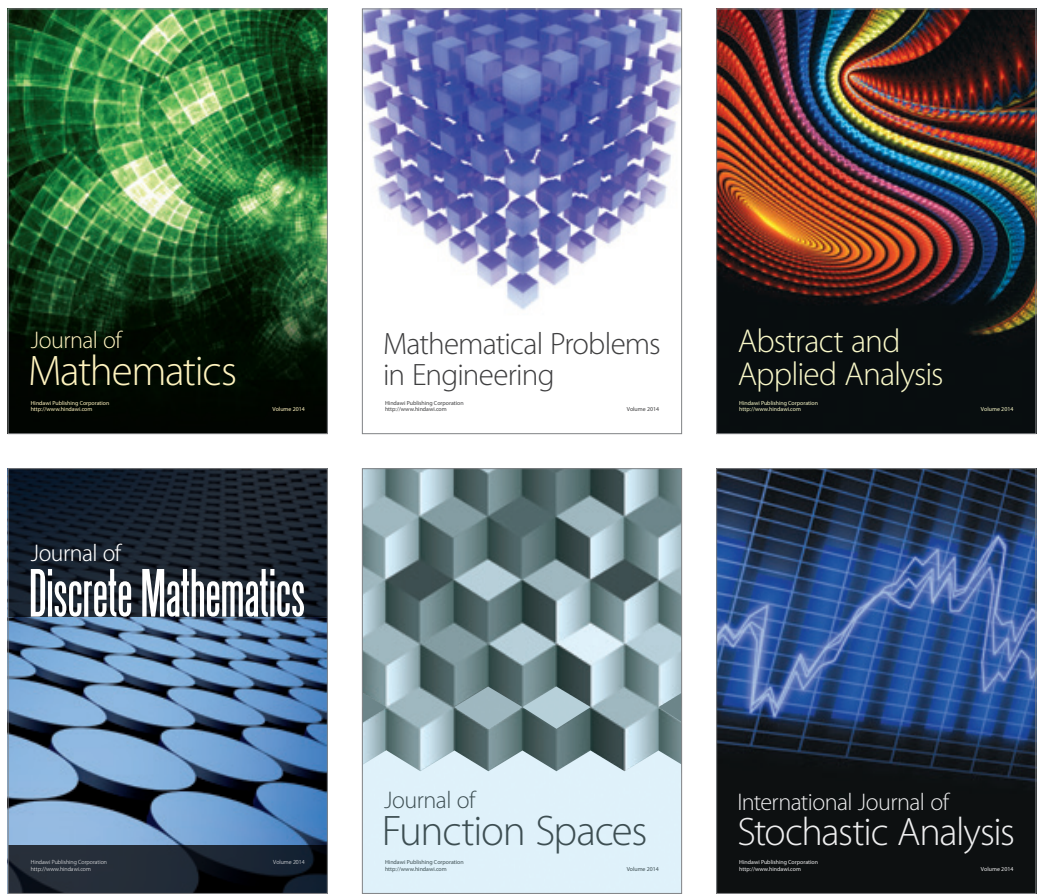

Journal of

Function Spaces

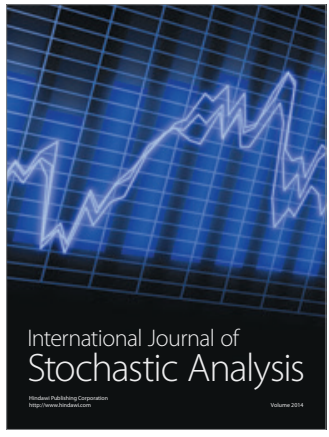

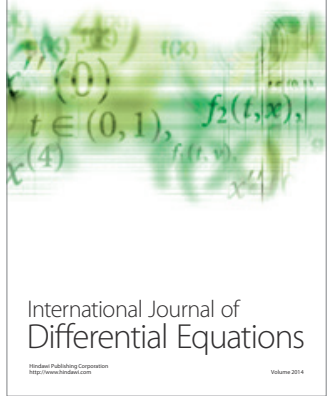
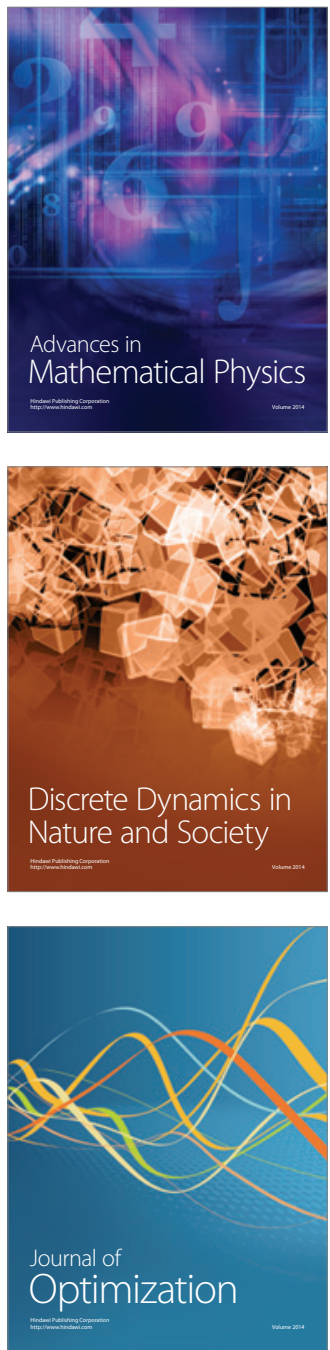